\title{
Assessment of fusimotor contribution to reflex reinforcement in humans ${ }^{1}$
}

\author{
DORIS BURG ${ }^{2}$, ALFRED J. SZUMSKI ${ }^{3}$, ALBRECHT STRUPPLER, \\ AND FRANCISCO VELHO
}

\author{
From the Neurological Clinic, Technical University of Munich, Munich, West Germany
}

SYNOPSIS The contribution of the fusimotor system to reflex reinforcement such as the Jendrassik $\stackrel{\varnothing}{\varnothing}$ manoeuvre was investigated by recording single unit activity with tungsten electrodes from muscle $s$ spindle afferent nerves in unanaesthetized normal human subjects. Muscle spindle afferent activity $\vec{\circ}$ was recorded before, during, and after the reinforcement test. When the leg muscles remained relaxed $\overrightarrow{\vec{\omega}}$ during the Jendrassik manoeuvre, spindle activity recorded in the tibial nerve was accelerated. Also in the median nerve, activity from muscle spindle afferent fibres was increased during a remote contraction of the ipsilateral quadriceps muscle. Comparing the time course of the phasic reflex rein forcement and the muscle spindle facilitation during the remote contraction, a marked after-effeet was recorded in both responses. Present results show an increased spontaneous muscle spindfe. activity in relaxed muscles during a remote muscle contraction, and provide evidence for the contribu tion of the fusimotor system to the enhancement of phasic reflexes by reinforcement manoeuvres $\frac{O}{\mathbb{D}} N$

The description of the Jendrassik handgrip (Jendrássik, 1883) has stimulated various theories concerning the mechanism involved in reflex reinforcement. It was originally assumed that reflex enhancement was due to a complete relaxation of lower extremity muscles when the patient's attention was diverted by the remote muscular effort of the handgrip. Hoffmann (1934) first proposed the hypothesis of reflex facilitation. Six years later, Sommer (1940) confirmed this hypothesis when he found that the $\mathrm{H}$-reflex which was known to bypass the muscle spindle was not enhanced by the Jendrassik manoeuvre. Taking the amplitude relationship of the electrically evoked H-reflex to the ankle jerk (T-reflex) as a measure of muscle spindle activity, he concluded that the facilitation of the phasic stretch reflex was due to muscle spindle activation. Paillard (1955a, b) confirmed these results. With the functional description of the gamma loop by Leksell (1945), Hunt and

\footnotetext{
1 Supported by the Deutsche Forschungsgemeinschaft.

2 Address for correspondence: Neurologische Klinik und Poliklinik. Technische Universität München, Möhlstrasse 28, 8000 München 80, W. Germany.

3 Alexander von Humboldt Fellow (Germany) on Educational Leave from the Medical College of Virginia, Virginia Commonwealth University, Richmond, Virginia.
}

Kuffler (1951a, b), Kuffler et al. (1951), a by Granit (1950), it was generally assumed the fusimotor activation produced reflex facilitatio 3 during the Jendrassik manoeuvre. Consequent璃. this test was used in man as a criterion of normat $\overrightarrow{0}$

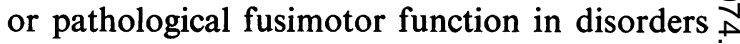
of the motor system (Buller and Dornhorst, 1957; Jung and Hassler, 1960; Ward, 1961; Hoffmann et al., 1962; Aranda et al., 1966; Hassler, 1972). Recently, however, many reports of H-reflex facilitation by contraction of remote $\stackrel{\mathbb{Q}}{\complement}$ muscles have appeared (Benson and Gedye, $\vec{\overrightarrow{ }}$ 1961; Landau and Clare, 1964; Gassel and 3 Diamantopoulos, 1964) which suggested that the results of Sommer (1940) were not valid in assuming that fusimotor activation played a role in reflex reinforcement. Furthermore, Landau and Clare (1964) reasoned that even when reflex facilitation is mediated by the gamma loop, the increased input by spindle afferent fibres is supposed to lower the threshold of motoneurones so that the number of motor units activated by an electrical stimulus to the peri- $\frac{T}{0}$ pheral nerve would be increased. Consequently, the question of reflex reinforcement cannot be $\mathrm{N}$ answered by an analysis of the relationship $N$ 012 
between $\mathrm{H}$ - and $\mathrm{T}$ - reflex facilitation, and the involvement of the fusimotor system remains unclear. In this regard, Clare and Landau (1964), using the differential nerve block technique (Matthews and Rushworth, 1957), showed that reflex reinforcement was more effective during fusimotor blockade, and concluded that reflex potentiation was mediated at the motoneurone level without the influence of the gamma loop. Currently, therefore, there is an argument against the hypothesis that reflex reinforcement is mediated by the gamma loop, but, since the results obtained under differential block cannot rule out fusimotor function under normal conditions, a new approach is required to the study of the mechanism of central facilitation by peripheral influences under normal conditions.

The method of percutaneous recording from single human nerve fibres with tungsten electrodes, developed and described in detail by Hagbarth and Vallbo (1967) and Vallbo and Hagbarth (1968), allows direct recording from afferent nerve fibres and an analysis of the proprioceptive inflow to the spinal cord under various conditions. As is widely known, in a relaxed muscle which is not influenced by external forces, the activity of muscle spindle afferent nerve fibres depends on muscle length and on the activity of corresponding fusimotor neurones. When muscle length is kept constant during contraction of remote muscles, the activity of muscle spindle afferent fibres is an indication of their fusimotor drive.

The purpose of this study was to reinvestigate the problem of reflex facilitation by means of single unit recording from muscle spindle afferent nerve fibres of a relaxed muscle during contraction of remote muscles to assess fusimotor function in reflex reinforcement under normal conditions. Evidence is presented that there is a marked increase of muscle spindle activity in a relaxed muscle during contraction of a remote muscle. Preliminary reports of these results have been presented by Burg et al. (1972) and Struppler et al. (1973).

\section{METHODS}

The results were obtained from 24 successful experiments in normal volunteer students who were unanaesthetized and remained awake throughout the experiment. Six of the subjects rested comfortably in a prone position with the foot extended over the edge of the examining table for recording from the tibial nerve and elicitation of the Achilles ankle jerk reflex. The foot was secured in a boot which was connected to a potentiometer allowing for constant monitoring of foot position and movement. The recording apparatus was sensitive to foot displacement of less than $0.5^{\circ}$. For the reinforcement test, the subjects were instructed to perform the Jendrassik handgrip manoeuvre. Eighteen subjects were tested in the supine position for investigation of the median nerve. The arm to be investigated was placed in a padded arm support in a natural position of approximately $70^{\circ}$ of shoulder abduction and with the forearm in supination. The support prevented extension of the fingers, wrist, and the entire upper extremity. For the reinforcement test of the forearm muscles, the subjects were instructed and trained to perform a slight isometric contraction of the remote ipsilateral quadriceps muscle without a coactivation of the forearm muscles. The effect of this remote muscular contraction on phasic stretch reflexes of forearm muscles was shown in six subjects.

Tungsten electrodes were used to record activity of single nerve fibres from the tibial or the median nerve in the popliteal space or in the middle of the upper arm. No anaesthesia was used for the percutaneous penetration of the electrode. The electrode had a shaft diameter of $0.2 \mathrm{~mm}$ and was coated with an epoxylite and had an impedance measured at $1 \mathrm{kHz}$ between 110 and $150 \mathrm{k} \Omega$ (Hagbarth and Vallbo, 1968; Vallbo and Hagbarth, 1968). A silver plate on the dorsal aspect of the hand or on the medial side of the lower leg was used as a reference electrode. Single unit action potentials were recorded with a conventional preamplifier which had a bandwidth of $100-10,000 \mathrm{~Hz}$ placed near the recording site.

The recording electrode was inserted manually into the skin and directed toward the location of the nerve. To avoid insertion of the electrode directly into the nerve and especially into cutaneous fascicles of the nerve, rectangular pulse electrical stimuli of $0.5 \mathrm{~ms}$ duration were applied as the electrode approached the nerve. Contraction of forearm flexor muscles or calf muscles on stimulation with $1.0 \mathrm{~V}$ or less without eliciting paraesthesias of the fingers or hand, or of the lower leg or foot were criteria for locating muscle fascicles in the nerve. Once located, stimulation was terminated, and the electrode was inserted manually into the nerve fascicle to isolate and record single action potentials from muscle receptor afferent fibres. The electromyograms (EMG) of remote muscle contraction from the quadriceps, biceps, pectoralis, or deltoid muscle and from the forearm flexor and calf muscles were recorded 


\section{A}

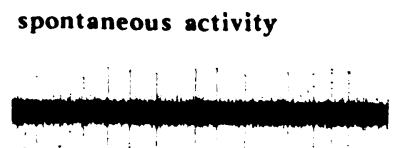

C

passive stretch

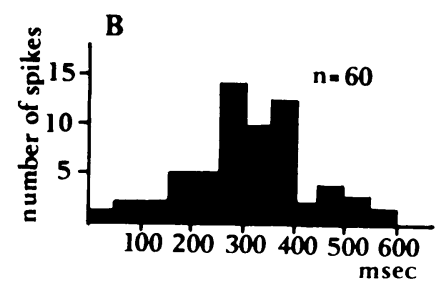

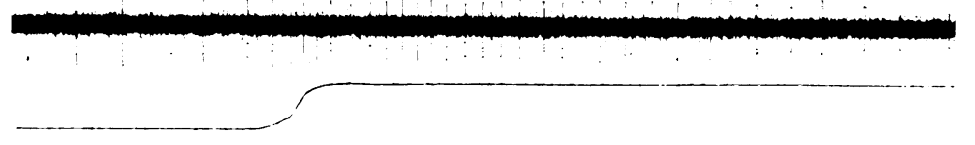

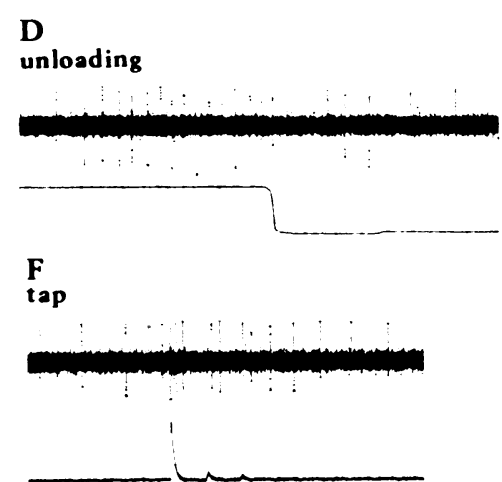

E phasic reflex

H

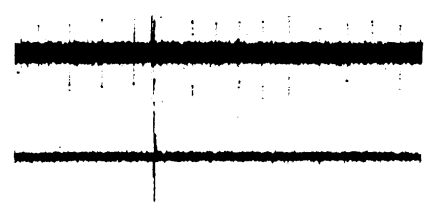

FIG. 1. Muscle receptor identification criteria.

\section{G twitch contraction}

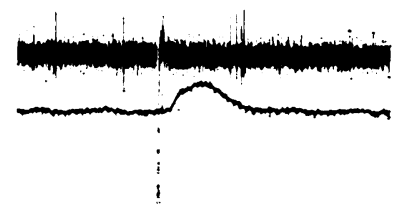

isometric contraction

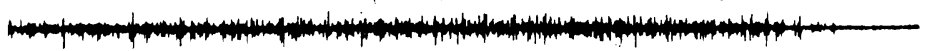

bipolarly with surface electrodes, and the nerve single unit potentials and the electromyograms were simultaneously displayed on a Tektronix 565 and a $5103 \mathrm{~N}$ oscilloscope and recorded by an Ampex 1300 tape recorder. An audio amplifier and a speaker were used to monitor action potential activity during electrode insertion and single unit activity. The recorded signals were replayed from the tape as permanent records on a Mingograph inkwriter. The muscle receptor of the isolated nerve fibre recorded in the tibial or median nerve was identified by several tests as illustrated in Fig. 1, which are similar to test criteria described by Vallbo $(1970 b, 1971)$. When action potentials were proved to originate from a muscle spindle of the calf muscles or forearm muscles, the subject was asked to perform the
Jendrassik handgrip or to contract the remote quadriceps muscle. When a coactivation of the muscle group in which the receptor was located was identified by observation or electromyographically, the test was excluded from the evaluation.

Twenty-four muscle spindle afferent fibres were identified and tested before, during, and after the reinforcement. The test could be repeated several times with most units.

\section{RESULTS}

MUSCLE RECEPTOR IDENTIFICATION When the electrode was located in a muscle fascicle of $\mathrm{N}$ the median or tibial nerve, afferent neural dis- 

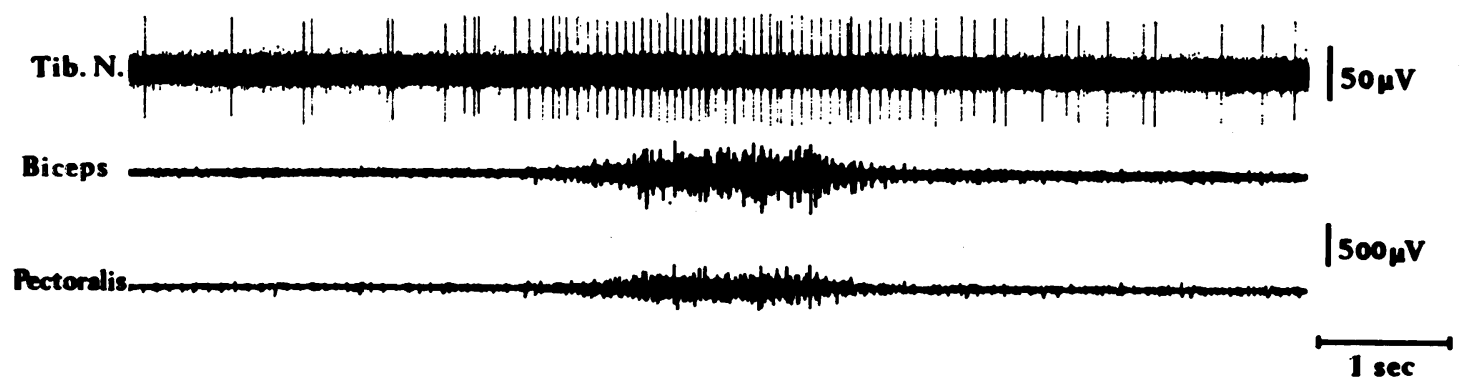

FIG. 2. Muscle spindle response during the Jendrassik manoeuvre. Upper trace, single unit potentials recorded from the tibial nerve; middle trace, biceps brachii EMG; lower trace, pectoralis major EMG.

charges could be obtained by mechanical stimuli applied to forearm or calf muscles, and with the electrode in this location usually no activity was obtained by stimuli applied to the skin innervated by the tibial or median nerve. Only recording sites giving exclusively afferent impulses on muscle stimulation were selected for evaluation. Single units were classified as originating from muscle afferent nerve fibres when they could be discharged with a short latency by mechanical deformation of the muscle. The receptor site was located where the lowest threshold of spike discharge to a manual muscle tap was found.

The criteria illustrated in Fig. 1A-H were used routinely to identify muscle spindles. The test shown in Fig. $1 \mathrm{G}$ could be applied only in recordings which proved to be stable for several minutes because the recording electrode was easily dislodged during the test. All muscle afferent nerve fibres described in this study had an irregular spontaneous activity which is illus- trated for one unit in Fig. 1A and in the interval histogram in Fig. 1B. When the muscle was stretched passively, the receptor showed a dynamic and a static sensitivity (Fig. 1C). After sudden unloading of the muscle, receptor activity stopped immediately and then restarted firing spontaneously (Fig. 1D). When a phasic reflex was elicited by a hammer tap, the afferent nerve fibre was activated with a short latency and the interspike interval was prolonged after the reflex EMG activity (Fig. 1E).

Mechanical pressure on the muscle activated a burst of neural activity corresponding to the force of the mechanical deformation. A slight muscle tap activated the receptor in a circumscribed part of the muscle. Figure 1F shows the afferent activity of a receptor which was located by manually tapping over the flexor digitorum superficialis muscle. A twitch contraction produced by an electrical stimulus applied to the muscle itself activated the muscle spindle during the relaxation phase of the twitch contraction

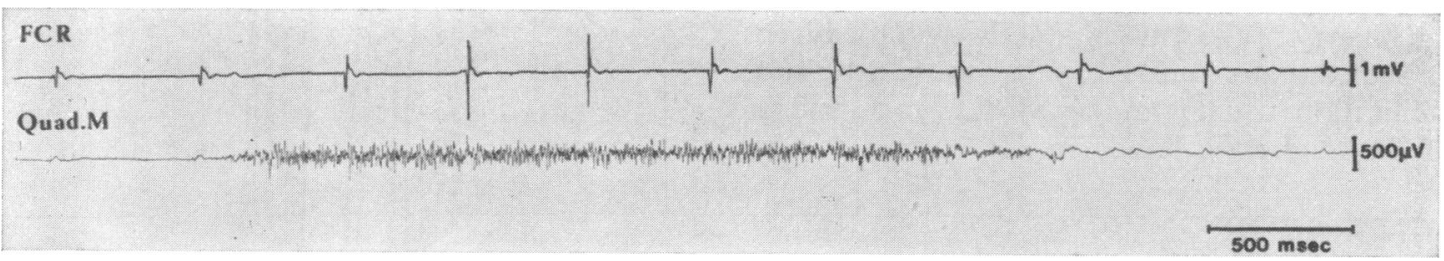

FIG. 3. Phasic reflex response during remote muscle contraction. Upper trace, flexor carpi radialis stretch reflex; lower trace, quadriceps muscle EMG. 
(Fig. 1G). When an isometric muscle contraction was performed there was an increase of spindle firing rate (Vallbo, 1971), and when the subject was asked to relax, a burst of activity occurred indicating the dynamic sensitivity of the receptor.

INFLUENCE OF JENDRASSIK MANOEUVRE ON ACTIVITY OF MUSCLE SPINDLES RECORDED IN TIBIAL NERVE In six subjects muscle spindle afferent nerve fibres were identified in the tibial nerve, and receptor spontaneous activity was recorded for several minutes while the subject remained relaxed. Since displacement of the foot as determined myographically was never recorded during the performance of the Jendrassik handgrip, the increased activity of the muscle spindle afferent nerve fibre was used as an indicator of gamma efferent drive during the test. In the spindle response illustrated in Fig. 2, the recording position was stable for about 20 minutes. The spontaneous activity of this receptor with the muscle at rest at an intermediate length was irregular and in the range of 0.5 to 2.5 impulses/s. After recording spontaneous activity, the subjects were instructed to perform the Jendrassik manoeuvre with slight or medium strength, and to keep their leg muscles relaxed during the experiment. When the subjects began to perform the Jendrassik manoeuvre, an immediate acceleration of muscle spindle activity was recorded in all trials. The latency was in the same range as the latency of the EMG recorded from the biceps, pectoralis or deltoid muscle. The latency of the muscle spindle facilitation in the tibial nerve ranged from $10 \mathrm{~ms}$ before the activation of the EMG to $80 \mathrm{~ms}$ after onset of EMG activity recorded in the arm muscles. Figure 2 illustrates a recording of the spontaneous activity originating from a muscle spindle and shows the facilitation during the Jendrassik manoeuvre. The latency of the muscle acceleration in this trial was about the same as the latency of the muscular activity in the biceps and pectoralis muscle. The muscle spindle facilitation usually outlasted the performance of the Jendrassik handgrip.

REFLEX REINFORCEMENT OF FOREARM FLEXOR MUSCLES In addition to the Jendrassik manoeuvre, most voluntary muscle activation is effective in reinforcing stretch reflexes of muscles which are not coactivated (Hoffmann, 1963). To assess this effect further for relaxed forearm $\bar{c}$ flexor muscles, phasic stretch reflexes of the $\stackrel{\infty}{=}$ flexor carpi radialis muscle elicited by a reflex $\vec{C}$ hammer tap applied to the tendon of this muscle at the wrist were tested before, during and after contraction of the ipsilateral quadriceps muscle. In all subjects the remote quadriceps contraction proved to be highly efficient in facilitating phasic stretch reflexes of the relaxed forearm flexor muscle. Figure 3 illustrates an example of a facilitation of the phasic stretch reflexes of the flexor carpi radialis muscle by contraction of a remote muscle. The reflex recorded by surface EMG electrodes over the flexor carpi radialis muscle never reached a

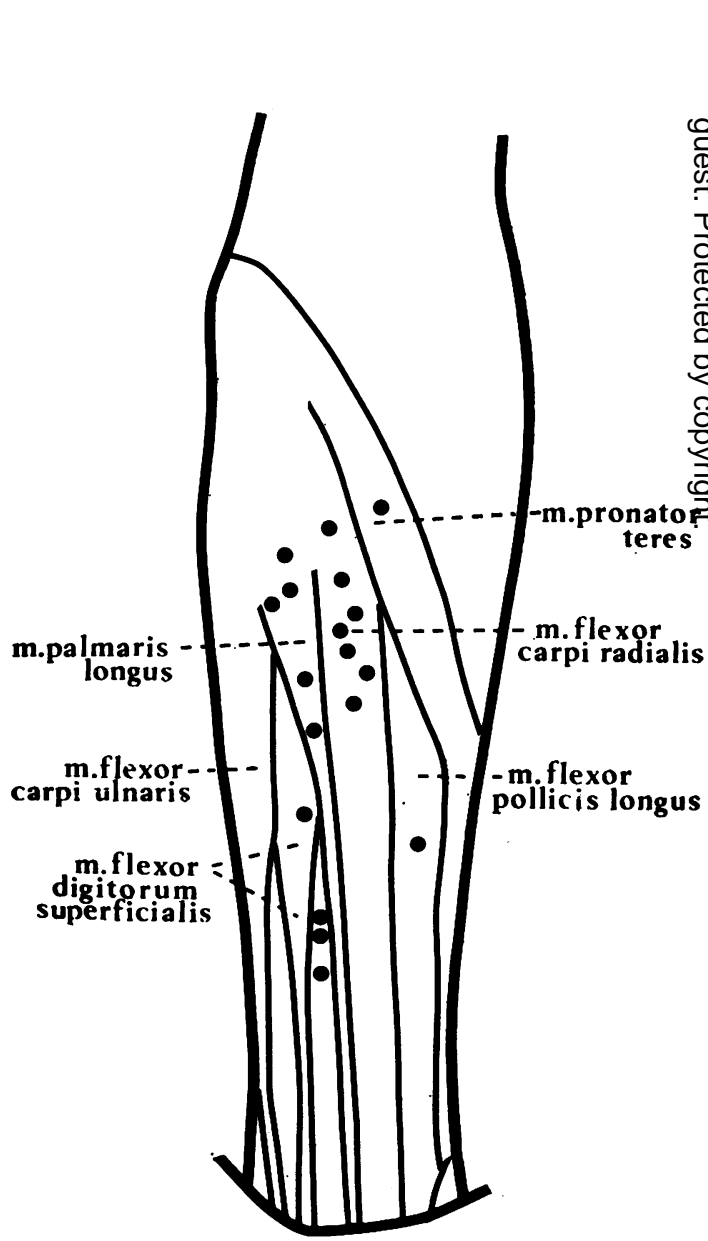

FIG. 4. Diagrammatic representation of forearm muscle receptor locations (O). 
height of $1.0 \mathrm{mV}$. During quadriceps contraction there was a clear enhancement of the reflex with recorded amplitudes in excess of $2.0 \mathrm{mV}$. The facilitation of the reflex outlasted the remote muscular contraction for about $500 \mathrm{~ms}$, sometimes even more than $2.0 \mathrm{~s}$. When the subjects were asked to strongly activate their quadriceps muscle, a coactivation of forearm muscles was very rarely detected by EMG activity. However, in these trials the reflex facilitation was not more marked than when no EMG activity in forearm muscles could be detected during contraction of the quadriceps muscle alone.

FOREARM MUSCLE SPINDLE FACILITATION BY REMOTE MUSCULAR CONTRACTION In order to monitor the effect of a remote muscular contraction-for example, a quadriceps contraction-. on the activity of muscle spindles in relaxed forearm muscles, 18 afferent nerve fibres coming from muscle spindles of forearm flexor muscles were identified in the median nerve. The location of the receptors was found by manually tapping over the muscle as indicated in Fig. 4 by the filled circles.

It could be shown that an increase of the firing frequency occurred in all fibres identified as coming from muscle spindles of forearm muscle with a slight isometric contraction of the ipsilateral quadriceps muscle. Figure 5A, B, and C illustrates a representative example of a muscle spindle facilitation in the median nerve by remote muscular contraction in a continuous recording from a spindle afferent nerve fibre. The receptor in this case was located in the palmaris longus muscle. The EMG of the ipsilateral quadriceps muscle was recorded simultaneously (second baseline of A, B, and C). During both muscular contractions muscle spindle afferent fibres are obviously activated. As can be seen in both contractions, especially in the second one, the firing frequency of the
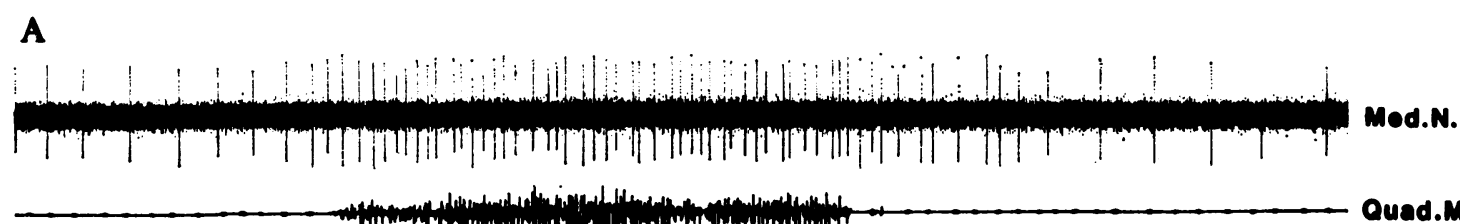
(n)

Quad.M.

\section{B}

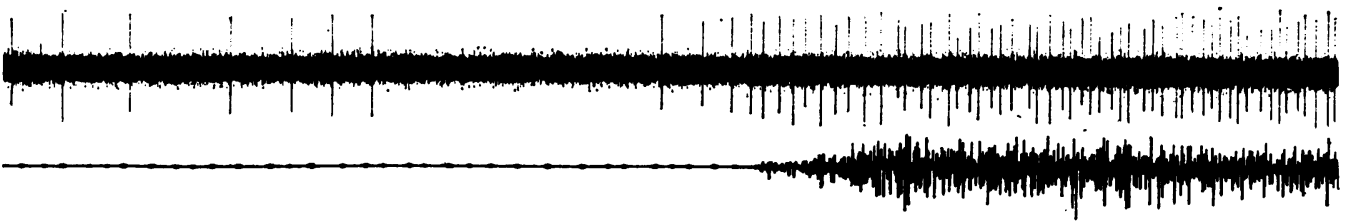

C
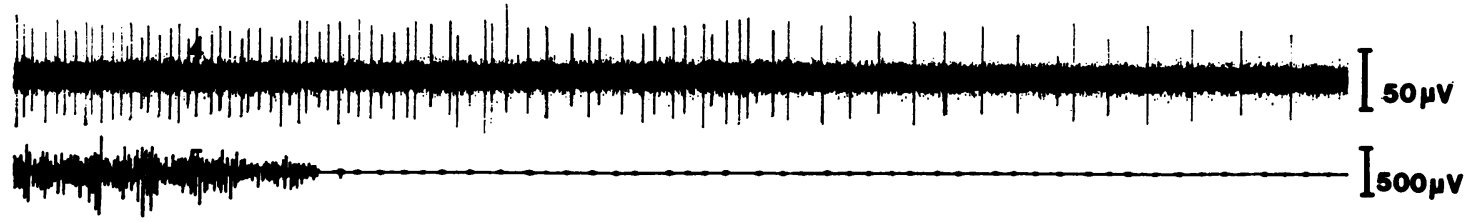

500 msec

FIG. 5. Muscle spindle response during remote muscle contraction. Upper traces, single unit potentials recorded from the median nerve; lower traces, quadriceps muscle EMG. Traces $\mathrm{A}, \mathrm{B}$, and $\mathrm{C}$ are continuous. 
afferent fibre increased with increasing EMG activity. It was found that muscle spindle afferent fibres which were silenced by passive shortening of the muscle began firing during a slight contraction of the quadriceps muscle. Also in this case the forearm, hand, and fingers were supported in the position of slight flexion of the hand and fingers so that no passive extension was possible during the reinforcement manoeuvre.

No obvious decrease of muscle spindle activity was observed when a remote contraction was maintained for more than $6.0 \mathrm{~s}$, unless the EMG activity of the remote muscle decreased. Usually there was an increase of receptor activity at the beginning or even shortly before the recorded contraction, with muscle spindle facilitation corresponding to the increasing remote muscular activity. With decreasing remote muscular activity, usually a reduction of muscle spindle activity was recorded. However, before the original receptor firing frequency was resumed after the relaxation of the quadriceps muscle, a marked after-effect was frequently recorded, as seen in both muscular contractions in Fig. 5 . This after-effect was variable and usually lasted at least $500 \mathrm{~ms}$. Only one muscle spindle, which was spontaneously active before the manoeuvre was silenced completely for about $1.0 \mathrm{~s}$ immediately after the reinforcement manoeuvre. Under high gain of the EMG, the latency between the beginning of the EMG of the quadriceps muscle and the activation of neural afferent activity in the median nerve was measured. In most cases, the latency ranged between zero and $60 \mathrm{~ms}$. In a few trials, muscle spindles of forearm muscles were activated before the EMG activity was recorded from the quadriceps muscle.

\section{DISCUSSION}

An obvious facilitation of muscle spindle activity in relaxed muscles during contraction of remote muscles has been shown in recordings from single muscle spindle afferent nerve fibres in normal subjects. This response was well marked from spindles in the calf muscles when the Jendrassik handgrip was performed, and it was also well marked in muscle spindles recorded from the forearm flexor muscles when leg muscles were contracted. The response to remote muscular contraction is known clinically to increase monosynaptic stretch reflexes not only $\underset{\mathbb{D}}{Z}$ with the classical Jendrassik manoeuvre (Jen- o drássik, 1883), but also with almost any muscu- @ lar effort (Hoffmann, 1963).

During recording of activity arising from 0 muscle spindles in the tibial and in the median nerve, every effort was exerted to ensure by observation, myographically, and by the position. of the limb that the length of the muscle in which the receptor was located was maintained constant throughout the experiment. Especially $\frac{C}{0}$ stretch of the muscle in which the receptor was $\frac{\bar{m}}{\bar{m}}$ located was excluded during recording in $\mathbb{\otimes}$ the median nerve by firm support of the dorsal aspect of the forearm, hand, and fingers which $\overrightarrow{ }$ did not allow an extension in the corresponding joints and, during recording in the tibial nerve, stretch of the calf muscles was excluded by myographically monitoring the foot position. Furthermore, the firing pattern of the spindle recept excluded the possibility that the increased firin frequency of the spindle during the remote con $\overrightarrow{0}$ traction was due to a slight passive stretch of the $\overrightarrow{\mathrm{N}}$ muscle in which the receptor was located, since $\frac{1}{2}$ 을 passive stretch in all units was evidenced by $\frac{\mathrm{Q}}{\mathrm{g}} \mathrm{C}$ dynamic burst at the beginning of the stretch, 0 slow adaptation during the maintained stretch and an immediate cessation of spindle activit when the stretch was released (Fig. 1C, D). minimal passive muscle stretch never caused $\vec{a}$ marked increase in the static spindle activity. In contrast, during a slight remote muscle contraction, muscle spindle activity was facilitated without a dynamic burst at the beginning of the contraction and the receptor did not show adaptation during the course of the remote contraction. Spindle facilitation in the relaxed muscle usually outlasted the remote muscle contraction and returned slowly to the original firing frequency (Fig. 2, Fig. 5). Consequently, it is concluded that the increased muscle spindle activity in relaxed muscles during a remote muscle contraction is due to a gamma efferent drive. However, on the basis of the present results, it is not possible to decide if the facilitation is specifically due to a static or to a dynamic gamma efferent drive, or to an activation of both gamma systems, since both are known to increase $\frac{T}{0}$ muscle spindle sensitivity and firing rate with the muscle at a constant length (Lewis and Proske, o 1972). 
It has been suggested in recent studies by Hagbarth and Vallbo (1968) and by Vallbo $(1970 \mathrm{a}, \mathrm{b})$ that a voluntary contraction of forearm or calf muscles also involves the gamma efferent system and produces a spindle facilitation. In order to minimize the possibility of coactivation of the muscles in which the receptor was located when the remote muscle was contracted, all subjects were trained to perform the reinforcement manoeuvre without any clinically detectable coactivation of forearm or calf muscles, and in eight subjects this was verified electromyographically. In all these experiments a well marked facilitation of muscle spindles could be recorded without extrafusal muscle activation.

In order to present evidence for the suggestion that the muscle spindle facilitation observed in this study is a basic mechanism underlying the phenomenon of reflex reinforcement, the time course of muscle spindle facilitation was compared with the time course of reflex reinforcement. For both reinforcement responses, a short latency and a marked after-effect lasting between 0.5 and $3.0 \mathrm{~s}$ was usually observed. For the reflex reinforcement by the Jendrassik handgrip the short latency (Buller and Dornhorst, 1957) and the after-effect (Struppler and Preuss, 1959) are well known, and the enhancement of forearm reflexes which appears immediately with a remote contraction of leg muscles and outlasts the contraction as seen in Fig. 3, is shown in this study. Spindle facilitation with a short latency and an obvious after-effect was observed in most of the trials in muscle spindles recorded from the tibial and the median nerve. The finding of a similar time course in both responses further supports the suggestion that the fusimotor drive is a basic mechanism of reflex reinforcement.

In contrast with the results of this study, the hypothesis that the fusimotor system is not a significant part of the pathway in reflex reinforcement was put forward by Clare and Landau (1964). These authors found T- and H-reflex reinforcement enhanced in experiments using the differential procaine block technique. The results suggested that the reinforcement had a facilitatory effect on alpha motoneurones, but under their experimental conditions they cannot exclude that some gamma efferent fibres were intact and could have been responsible for the reinforcement effect, especially when the control response is minimal. If the increased reinforcement response were due to blocking the gamma system, an inhibition of this system during the manoeuvre could account for a less marked reflex enhancement with intact gamma fibres. In the present results, however, an inhibition of muscle spindle activity was never recorded.

The results from the present experiments and those of Clare and Landau (1964) could possibly confirm the facilitatory effect if it were found to be due to a pure static gamma drive. Under these conditions of selective gamma efferent blockade, the dynamic sensitivity of muscle spindles could be increased and a facilitation of alpha motoneurones by the reinforcement manoeuvre would further increase reflex excitability. However, the increase of spontaneous spindle activity with a pure static gamma drive supposedly lowers the threshold of alpha motoneurones so that reflex excitability would be increased rather than decreased.

Present results support the concept of Sommer (1940) that an increased muscle spindle activity accounts at least partly for the enhancement of reflex excitability during reinforcement manoeuvres. The same conclusion was drawn by Burke et al. (1972) when testing the effect of the Jendrassik manoeuvre on the tonic vibration reflex. These authors found that the tonic vibration reflex increased during the performance of the Jendrassik handgrip, and especially when the muscle was at a shortened length and the muscle spindles were unloaded. They concluded from their results that an increased fusimotor drive compensated for the unloaded muscle spindles thus increasing the sensitivity of the primary endings of muscle spindles and thereby increasing the reflex response to vibration.

The present experiments probably include only recordings from afferent fibres arising from spindle primary receptor endings (Burg et al., 1973). Three muscle spindle receptor identification criteria were recognized in all 24 receptors. The criteria were: (1) an irregular spontaneous activity which has been shown to be more marked in group Ia than in group II afferent fibres (Matthews and Stein, 1969); (2) the receptors had a definite dynamic sensitivity as shown by passive stretch (Matthews, 1963); and (3) they all stopped firing immediately when the muscle 
was suddenly unloaded (Struppler and Erbel, 1971), a response shown by Cooper (1961) to be typical for spindle primary endings. In studies investigating the influence of the Jendrassik manoeuvre on receptor neural activity in the peroneal and tibial nerve, Hagbarth and Vallbo (1968) did not find an activation when the leg muscles remained relaxed. Tapping and vibration of the muscle were utilized as receptor identification tests. Their finding suggests that not all muscle spindles are facilitated by a remote voluntary effort. However, the muscle spindle activity recorded in 24 identified units of this study is in favour of the importance of this mechanism of facilitating the reflex excitability of the motor system during a restricted voluntary contraction.

With regard to the mechanism of reflex facilitation, present results cannot rule out that during a remote muscular contraction there might be, in addition to a fusimotor drive, a direct influence on alpha motoneurones. This suggestion is supported by the results of Clare and Landau (1964) in the reinforcement response during differential block, and by the studies of Struppler and Preuss (1959) and of Gassel and Diamantopoulos (1964) stressing a different time course of the reinforcement effect on the $\mathrm{T}$ - and $\mathrm{H}$-reflex. In both reflex studies no after-effect was seen when the H-reflex was tested, and, in contrast, sometimes even a depression of the H-reflex was seen after a reinforcement. This effect on the $\mathrm{H}$-reflex cannot be explained by our findings, whereas the effect on the T-reflex can readily be explained by an increased fusimotor drive for which our results present evidence.

The finding of an increased fusimotor drive during reflex reinforcement manoeuvres in normal subjects does not yet justify theories about pathological states in which reflex reinforcement is less marked. More details concerning the specific influence of reinforcement on the dynamic or static fusimotor drive are needed for further interpretation, and direct investigation of the reinforcement effect on muscle spindle activity in pathological states might further contribute to the understanding of the pathomechanism of hyperactive states.

The authors acknowledge the capable technical assistance of Miss Mechthild Haberkamp.

\section{REFERENCES}

Aranda, C. L., Rocamora, R. G., Asenjo, G. A., Chiorino, R. R., and Donoso, Y. P. (1966). Alpha and gamma motor systems in extrapyramidal diseases. Confinia Neurologica, 27, 230-233.

Benson, A. J., and Gedye, J. L. (1961). Some supraspinal factors influencing generalised muscle activity. In Proceedings of a Symposium on Skeletal Muscle Spasm, held $\overline{\overline{0}}$ under the auspices of Riker Laboratories, 1961, pp. 31-50. Franklyn Ward, and Wheeler: Leicester.

Buller, A. J. (1957). The ankle-jerk in early hemiplegia. Lancet, 2, 1262-1263.

Buller, A. J., and Dornhorst, A. C. (1957). The reinforcement of tendon-reflexes. Lancet, 2, 1260-1262.

Burg, D., Struppler, A., and Velho, F. (1973). Assessment of $\overline{\bar{A}}$ fusimotor function in reflex reinforcement. (Abstract.) Pflügers Archiv, 339, Suppl., R75.

Burg, D., Szumski, A. J., Struppler, A., and Velho, F. (1973). ஸి Afferent and efferent activation of human muscle receptors $\overrightarrow{0}$ involved in reflex and voluntary contraction. Experimental Neurology, 41, 754-768.

Burke, D., Andrews, C. J., and Lance, J. W. (1972). Tonic $\vec{\omega}$ vibration reflex in spasticity, Parkinson's disease, and normal subjects. Journal of Neurology, Neurosurgery, and Psychiatry, 35, 477-486.

Clare, M. H., and Landau, W. M. (1964). Fusimotor function. Part 5. Reflex reinforcement under fusimotor block normal subjects. Archives of Neurology (Chic.), 10, 123127.

Cooper, S. (1961). The responses of the primary and secone्? $\vec{N}$ ary endings of muscle spindles with intact motor innerves 응 tion during applied stretch. Quarterly Journal of Expeft mental Physiology, 46, 389-398.

Gassel, M. M., and Diamantopoulos, E. (1964). T迟 Jendrassik maneuver: 1 . The pattern of reinforcement of monosynaptic reflexes in normal subjects and patients wigh $\frac{(D)}{3}$ spasticity or rigidity. Neurology (Minneap.), 14, 555-560

Granit, R. (1950). Reflex self-regulation of muscle contrāition and autogenetic inhibition. Journal of Neurophysiolog 13, 351-372.

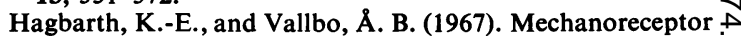
activity recorded percutaneously with semi-microelectrodes in human peripheral nerves. Acta Physiologica Scandinavica, 69, 121-122.

Hagbarth, K.-E., and Vallbo, A. B. (1968). Discharge characteristics of human muscle afferents during muscle stretch and contraction. Experimental Neurology, 22, 674-694.

Hassler, R. (1972). Physiopathology of rigidity. In Parkinson's Disease, Rigidity, Akinesia, Behavior. Vol. 1, pp. 19- $\overline{\bar{O}}$ 45. Edited by J. Siegfried. Huber: Bern.

Hoffmann, P. (1934). Die physiologischen Eigenschaften der Eigenreflexe. Ergebnisse der Physiologie, Biologischen Chemie und Experimentellen Pharmakologie, 36, 15-108.

Hoffmann, P. (1963). Der Jendrassiksche Kunstgriff und die Aufklärung seiner physiologischen Bedeutung. Münchener Medizinische Wochenschrift, 105, 1629-1634.

Hofmann, W. W., Angel, R. W., Seil, F. J., and Schear, H. E. 응 (1962). Reflex re-enforcement in parkinsonism. Neurology (Minneap.), 12, 440-442.

Hunt, C. C., and Kuffler, S. W. (1951a). Further study of efferent small-nerve fibres to mammalian muscle spindles.음 Multiple spindle innervation and activity during contraction. Journal of Physiology, 113, 283-297.

Hunt, C. C., and Kuffler, S. W. (1951b). Stretch receptor dis-을 charges during muscle contraction. Journal of Physiology, $N$ 113, 298-315.

Jendrássik, E. (1883). Beiträge zur Lehre von den Sehnen-N 
reflexen. Deutsches Archiv für Klinische Medizin, 33, 177199.

Jung, R., and Hassler, R. (1960). The extrapyramidal motor system. In Handbook of Physiology, section 1, vol. 2, pp. 863-927. American Physiological Society: Washington.

Kuffler, S. W., Hunt, C. C., and Quilliam, J. P. (1951). Function of medullated small-nerve fibers in mammalian ventral roots: efferent muscle spindle innervation. Journal of Neurophysiology, 14, 29-54.

Landau, W. M., and Clare, M. H. (1964). Fusimotor function. Part 4. Reinforcement of the $\mathbf{H}$ reflex in normal subjects. Archives of Neurology and Psychiatry (Chic.), 10, 117122.

Leksell, L. (1945). The action potential and excitatory effects of the small ventral root fibres to skeletal muscle. Acta Physiologica Scandinavica, 10, Supplement 31, 1-84.

Lewis, D. M., and Proske, U. (1972). The effect of muscle length and rate of fusimotor stimulation on the frequency of discharge in primary endings from muscle spindles in the cat. Journal of Physiology, 222, 511-535.

Matthews, P. B. C. (1963). The response of de-efferented muscle spindle receptors to stretching at different velocities. Journal of Physiology, 168, 660-678.

Matthews, P. B. C., and Rushworth, G. (1957). The selective effect of procaine on the stretch reflex and tendon jerk of soleus muscle when applied to its nerve. Journal of Physiology, 135, 245-262.

Matthews, P. B. C., and Stein, R. (1969). The regularity of primary and secondary muscle spindle afferent discharges. Journal of Physiology, 202, 59-82.

Paillard, J. (1955a). Réflexes et Régulations d'Origine Proprioceptive chez l'Homme. Arnette: Paris.

Paillard, J. (1955b). Analyse électiophysiologique et com- parison, chez l'homme, du réflexe de Hoffmann et du réflexe myotatique. Pflügers Archiv, 260, 448-479.

Sommer, J. (1940). Periphere Bahnung von Muskeleigenreflexen als Wesen des Jendrassikschen Phänomens. Deutsche Zeitschrift für Nervenheilkunde, 150, 249-262.

Struppler, A., Burg, D., Velho, F., and Szumski, A. J. (1973). Fusimotor contribution to the reflex reinforcement in the Jendrassik maneouvre. (Abstract.) J. of Electroencephalography and Clinical Neurophysiology, 34, 826.

Struppler, A., and Erbel, F. (1972). Analysis of proprioceptive excitability with special reference to the "unloading reflex'. In Neurophysiology Studied in Man, pp. 298-304. Edited by G. G. Somjen. International Congress Series No. 253. Excerpta Medica: Amsterdam.

Struppler, A., and Preuss, R. (1959). Untersuchungen über periphere und zentrale Faktoren der Eigenreflexerregbarkeit am Menschen mit Hilfe des Jendrassikschen Handgriffes. Pflügers Archiv für die gesamte Physiologie des Menschen und der Tiere, 268, 425-434.

Vallbo, Å. B. (1970a). Discharge patterns in human muscle spindle afferents during isometric voluntary contraction. Acta Physiologica Scandinavica, 80, 552-566.

Vallbo, A. B. (1970b). Slowly adapting muscle receptors in man. Acta Physiologica Scandinavica, 78, 315-333.

Vallbo, $\AA$. B. (1971). Muscle spindle response at the onset of isometric voluntary contractions in man. Time difference between fusimotor and skeletomotor effects. Journal of Physiology, 318, 405-431.

Vallbo, Å. B., and Hagbarth, K.-E. (1968). Activity from skin mechanoreceptors recorded percutaneously in awake human subjects. Experimental Neurology, 21, 270-289.

Ward, A. A., Jr (1961). Physiological mechanisms in parkinsonism. Revue Canadienne de Biologie, 20, 345-350. 